\title{
Comparison of climate reconstruction methods, modeling, and data synthesis approaches
}

\author{
Joëlle Gergis', A.M. Lorrey², N.J. Abram³ ${ }^{3}$ B.J. Henley', S.J. Phipps ${ }^{4}$, K.M. Saunders ${ }^{5}$ and workshop participants ${ }^{6}$
} 4th Australasia2k workshop, Auckland, New Zealand, 27-29 October 2015

This workshop, held at the National Institute of Water and Atmospheric Research (NIWA) in Auckland, reviewed research progress and identified potential contributions from the Australasian research community to Phase 2 of the PAGES 2k Network. The workshop included specialists in data collection, data synthesis, climate reconstruction and climate modeling, with increased involvement from lake-sediment and speleothem communities. This multidisciplinary group generated many insightful and collegial discussions that resulted in a highly productive workshop and the establishment of new collaborations.

The presentations showcased developments in data synthesis, climate modeling and new record development. Helen McGregor discussed a low-resolution sea surface temperature composite by the Ocean2k group (McGregor et al. 2015). Nerilie Abram presented the PAGES $2 \mathrm{k}$ Consortium effort focused on the onset of Industrial-era warming across oceans and continents. Mandy Freund presented a new Australian precipitation field reconstruction spanning the last millennium.

Hamish McGowan showed a 2100-year temperature reconstruction using a speleothem from New South Wales, Australia. Chris Moy linked high-accumulation lake records from southern South America and the Auckland Islands to changes in the Southern Hemisphere westerly winds. Ben Henley presented an Interdecadal Pacific Oscillation reconstruction from Pacificwide paleoclimate data using the new Tripole Mode Index (Henley et al. 2015).
Work connecting proxy data and climate model simulations by Steven Phipps and Duncan Ackerley showcased data assimilation for Southern Hemisphere records using CSIRO $\mathrm{Mk} 3 \mathrm{~L}$ model simulations, and potential for conducting paleoclimate experiments using the Australian Centre for Water and Climate Research's ACCESS model.

Bronwyn Dixon described the new Australasian "low resolution" database, which includes 536 non-annually resolved records from Australia and Indonesia. The highest quality records were identified and preliminary results presented. Stuart Browning gave a presentation on a new paleoclimate data and climate model-data assimilation method (Browning and Goodwin 2015) to develop a 1000-year reanalysis product called "PaleoR" (http://climatefutures. mq.edu.au/research/themes/marine/paleor/). Following this, Drew Lorrey showcased NIWA's Past Interpretation of Climate Tool (PICT; http://pict.niwa.co.nz), which applies modern analogues from reanalysis data to understand past circulation modes from paleoclimate data (Lorrey et al. 2013). A comparison of results derived from the PaleoR and PICT methods for the 1450-1850 CE period is shown in Figure 1. Lively discussions led by lan Goodwin considered developing guidelines for future record collection in the Australasian region, framed around understanding regional and hemispheric climate dynamics.

Marcus Vandergoes presented progress developing high-resolution lake sediment records from New Zealand, followed by Krystyna
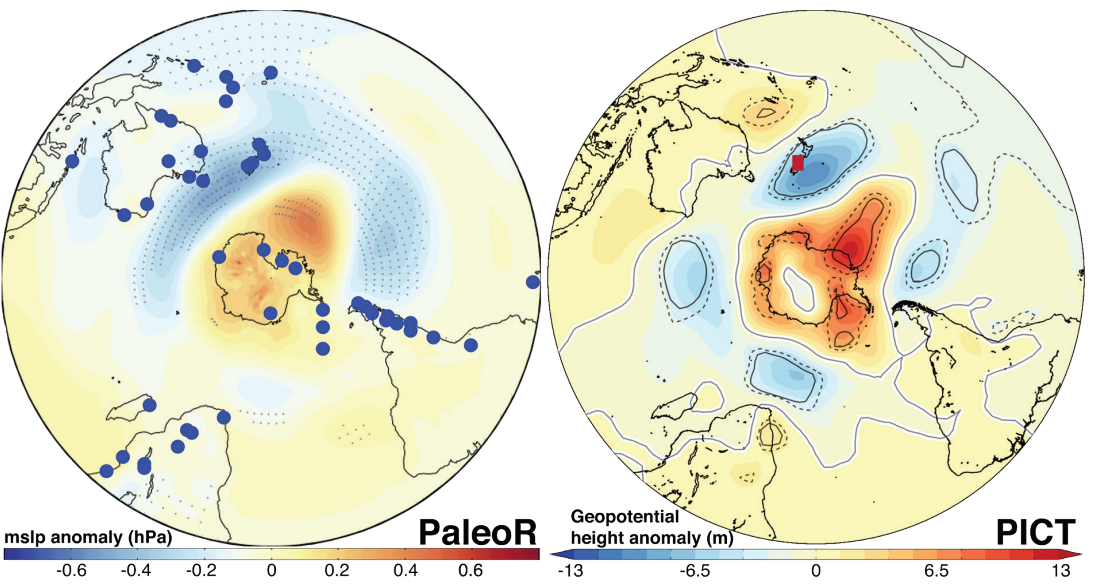

Figure 1: Southern Hemisphere spatial fields for austral summer near-surface atmospheric circulation (1450-1850 CE) based on PaleoR and PICT. PaleoR is based on a diverse range of proxy data from across the globe, while PICT is based on average paleotemperatures from 22 Southern Alps glaciers in New Zealand. Proxy data are independent and derived spatial fields are based on different datasets (PaleoR=climate model; PICT=modern reanalysis). There are broad similarities, along with regional confidence for different parts of the Southern Hemisphere.
Saunders' precipitation reconstruction from Tasmanian lake sediments based on scanning visible reflectance spectroscopy. An interactive poster session highlighted progress on the climatic interpretation of Western Australian speleothem records by Pauline Treble; a new borehole temperature reconstruction for eastern Australia by Suman Asadusjjaman; sedimentary charcoal records from Pacific Islands by Matthew Prebble; and a new high-resolution Southern Hemisphere westerly winds reconstruction using sub-Antarctic lake sediments by Krystyna Saunders.

There was extensive discussion on regional "best practice" standards for proxy metadata reporting and compatibility with the LiPD framework (McKay and Emile-Geay 2016), existing geochronology protocols, ideas for new record collection that support a new metadata template, and a proposed article on this critical topic.

The workshop identified two contributions on regional hydroclimate synthesis papers, led by early-career researchers Mandy Freund and Bronwyn Dixon, for the planned PAGES $2 \mathrm{k}$ synthesis special issue. A proposed table of contents for a regional special issue for Climate of the Past was generated to showcase the diversity of new research being developed by the Aus $2 \mathrm{k}$ community. The proposed submission timeframe is expected to span September 2016 to June 2017. Please contact a steering committee member if you are interested in contributing.

\section{AFFILIATIONS}

'School of Earth Sciences, University of Melbourne, Australia

${ }^{2}$ National Institute of Water and Atmospheric Research (NIWA), Auckland, New Zealand

Research School of Earth Sciences, Australian National University, Canberra, Australia

${ }^{4}$ Institute for Marine and Antarctic Studies, University of Tasmania, Hobart, Australia

${ }_{5}^{5}$ Australian Nuclear Science and Technology Organisation (ANSTO), Sydney, Australia

Www.pastglobalchanges.org/calendar/ all-events/127-pages/1490-4th-aus2k-wshop

\section{CONTACT}

Joelle Gergis: jgergis@unimelb.edu.au

REFERENCES

Henley B et al. (2015) Clim Dyn 45: 3077-3090

Lorrey A et al. (2013) Clim Dyn 42: 3039-3060

McGregor HV et al. (2015) Nature Geosci 8: 671-677

McKay NP, Emile-Geay J (2016) Clim Past 12: 1093-1100 